\title{
The Efficacy of Integrating Spirituality into Prenatal Care on Pregnant Women's Sleep: A Randomized Controlled Trial
}

\author{
Nahid Moradi $\left(\mathbb{D},{ }^{1}\right.$ Azam Maleki $\mathbb{D}^{2},{ }^{2}$ and Saeedeh Zenoozian $\mathbb{D}^{3}$ \\ ${ }^{1}$ School of Nursing and Midwifery, Zanjan University of Medical Sciences, Zanjan, Iran \\ ${ }^{2}$ Social Determinants of Health Research Center, Zanjan University of Medical Sciences, Zanjan, Iran
}

${ }^{3}$ Department of Mental Health, Zanjan University of Medical Sciences, Zanjan, Iran

Correspondence should be addressed to Azam Maleki; malekia@zums.ac.ir

Received 18 November 2021; Revised 31 December 2021; Accepted 20 January 2022; Published 3 February 2022

Academic Editor: Ming Chin Lan

Copyright (c) 2022 Nahid Moradi et al. This is an open access article distributed under the Creative Commons Attribution License, which permits unrestricted use, distribution, and reproduction in any medium, provided the original work is properly cited.

Objective. The study is aimed at determining the efficacy of spiritual content counselling on improving the sleep quality and insomnia severity of pregnant women. Methods. This randomized controlled trial was carried out on 40 pregnant women recruited at five health centres of Abhar, Iran, 2020. The eligible women were allocated into two intervention and control groups according to the randomized blocking method. Group counselling with spiritual content was carried out in eight sessions at 16 to 20 weeks of gestation. The control group only received routine care. Data were collected using the Pittsburgh Sleep Quality Index (PSQI) and the Insomnia Severity Index (ISI) questionnaires in three stages, before the intervention, at 28, and 36 weeks of gestation. Statistical analysis was performed using repeated measure ANOVA test, chi-square, and independent $t$-tests. $P<0.05$ was considered significant statistical level. Results. In the counselling group, the mean (SD) of a total score of sleep quality before the intervention was 9.45 (2.30) which decreased to 5.40 (1.56) in 36 weeks of gestational age, while in the control group was increased from 9.26 (2.15) to 11.47 (1.54). After the intervention based on the repeated measure ANOVA test, the mean total score of the insomnia severity, sleep quality, and its components decreased statistically in the second and third trimesters compared to the first trimester in the intervention group compared to the control group $(P=0.001)$. Conclusion. The results showed that counselling with spiritual content could effectively ameliorate sleep quality and reduce insomnia severity in pregnant women. It seems that the approach is an acceptable basis to design intervention programs in this field that can be considered by midwives. Clinical Trial Registry and Registration Number. The study was registered at the Iranian Registry of Clinical Trials under the IRCT20150731023423N15.

\section{Background}

One of the most common complaints during pregnancy is to have sleep disorders, such as insomnia, frequently waking up at night, high drowsiness during the day, mood swings, and unusual feelings during sleep [1]. In one review, more than $80 \%$ of pregnant women had sleep disorders [2]. A person's ability to fall asleep and the positive feeling after waking up indicate an ideal sleep quality [3]. The sleep quality varies in the different trimesters of pregnancy. Sleep disorders during pregnancy can increase unfortunate consequences, such as preeclampsia, preterm labor, miscarriage, urgent cesarean delivery, gestational diabetes, and postpartum depression [4-6].
To date, some studies have been conducted to improve sleep quality in pregnant women using a variety of pharmacological and nonpharmacological methods, each of which had different efficacy. The review of fourteen RCTs with 910 participants in Yan-Hua Lin's study shows that traditional Chinese medicines have significant effects on improving the Pittsburgh Sleep Quality Index [7]. In another study, Afrasiabian et al. show that the use of lemon verbena, an hour before bedtime for 4 weeks, could be improved significantly by the Pittsburgh Sleep Quality Index (PSQI) and Insomnia Severity Index (ISI) compared with the control group [8].

Today, religious beliefs are considered by many researchers as a treatment of mental and sleep disorders [9, 
10]. According to the religious beliefs in Persian medicine, listening to music has a positive effect on sleep quality by impressing the soul, particularly when it is played within special position [11].

If spiritual health is at risk, loneliness, anxiety, and loss of meaning in life will be likely developed. However, strengthening the spirituality in patients allows them to adapt effectively to their problems. Therefore, being connected with higher power and support from spiritual or religious sources can improve one's quality of life, control mental health disorders, and create interpersonal support $[10,12]$. In some studies, the use of spiritual counselling has been emphasized in the treatment of various aspects of pregnancy and childbirth, such as the stress during pregnancy and childbirth self-efficacy $[13,14]$. Spirituality is a key factor in promoting maternal and fetal health [13-15]; it plays an important role in the acceptance of pregnancy [16]. According to studies, spiritual counselling has been effective in improving sleep quality for coronary artery disease patients [9] and the elderly [10]. Nevertheless, there is an information gap in the efficacy of spiritual content counselling in improving sleep quality for women during pregnancy. Given the importance of culture and religion in many countries and the role of midwives in identifying the attitudes and spiritual beliefs of the individuals, the study is aimed at determining the efficacy of spiritual content counselling on improving the sleep quality and insomnia severity of pregnant women.

\section{Methods}

2.1. Setting and Participants. The present study was a randomized control trial to determine the efficacy of counselling with spiritual content on improving prenatal sleep quality and sleep intensity in 2020. The study setting included 5 community health centres in Abhar, and the research population included pregnant women referred to the centres. Abhar is a city in Zanjan Province, in the northwest of Iran. Abhar has 5 health centres, and sampling had been done in the centres.

The sample size was calculated based on the sleep quality variable in Malikzadegan's study [17] using the formula for calculating the sample size in two independent groups. With considering $95 \%$ confidence interval $(\mathrm{Z} 1-\alpha=1.96)$, the test power of $80 \%(Z 1-\beta=0.85)$, and the mean (standard deviation) of sleep quality in counselling group $\left(M_{1}=5.08\right.$, $\left.S_{1}=4.24\right)$ and $\left(M_{2}=8.31, S_{2}=1.86\right)$ in control groups, respectively, the total sample size was calculated to be 16 people. Due to the possibility of a $10 \%$ attrition in sample size, it increased to 20 in each group. Among 56 pregnant women evaluated by the first researcher, 40 women met the eligible criteria. The eligible pregnant women were assigned randomly to the two control and intervention groups based on the four-block design. In this way, six possible blocks will be assigned to group A (intervention) and group B (control). Then, the number of blocks was selected from the random number table until the study sample size reached. To ensure the concealment of the sequence of enrollment, we used the sequentially numbered, opaque sealed envelopes by a person who was not directly involved in the intervention.

The inclusion criteria were the age group of 18 to 35 years, the gestational age of 16-20 weeks, willingness to participate in the study, scoring more than 5 based on the Pittsburgh Sleep Quality Index (PSQI), and scoring less than 22 based on the General Health Questionnaire (GHQ-28). The exclusion criteria before randomization were consumption of sleeping pills, medical and obstetrics complications, and participation in a similar training program. There was no attrition in the study and after the interventions (Figure 1).

2.2. Procedure/Intervention. The spiritual content counselling was conducted in a place with a quiet and private setting for counselling in a health centre by the first author who is a midwife and familiar with group counselling under the supervision of a clinical psychologist. The content of counselling was developed by the research team based on the protocol of spiritual skills of Bolhari [18] and Asadi Zandi [19]. The number of the meeting was 8 sessions in two groups of 6 people and one group of 8 people. The meeting was held in 45-60 min for four following weeks (twice a week).

All the counselling sessions were attempted to use the principles and techniques of counselling to communicate effectively between the counsellor and participants. The counselling session was full of respect and intimacy and high self-confidence to provide an incredible opportunity for individuals to participate in group discussions. The content of each counselling session is listed in Table 1.

Data were completed by participants using the personal information questionnaire and the Pittsburgh Quality Sleep and the Insomnia Severity Index questionnaires in three stages, before the intervention, 28, and 36 weeks of gestational age.

2.3. Individual Information Questionnaire. The demographic questionnaire included age, level of education, employment status of the woman and her spouse, family income level, number of pregnancies, gestational age, number of abortions, pregnancy requirements, and residence status.

2.4. The Pittsburgh Sleep Quality Index (PSQI). The Pittsburgh Quality Sleep Questionnaire was used to measure sleep quality over the past month with seven components: subjective sleep quality, sleep latency, sleep duration, habitual sleep efficiency, sleep disturbances, use of sleeping medication, and daytime dysfunction. Scoring the questions on a Likert scale ranges from zero to three. The total score ranges from zero to 21 . People who score five or less are considered to have a good sleep quality. The reliability of the Persian version of the questionnaire was 0.65 . Furthermore, the content validity was acceptable $(\mathrm{CVI}=0.78$ and $\mathrm{CVR}=0.90)[20]$.

2.5. Insomnia Severity Index (ISI). The Insomnia Severity Index is a brief self-report tool that assesses patient's perception of the intensity of insomnia. This index has seven questions [21]. Scoring questions on 5 Likert scales ranges from zero (never) to 4 , so the score range is between 0 and 28 . 
CONSORT Flow Diagram

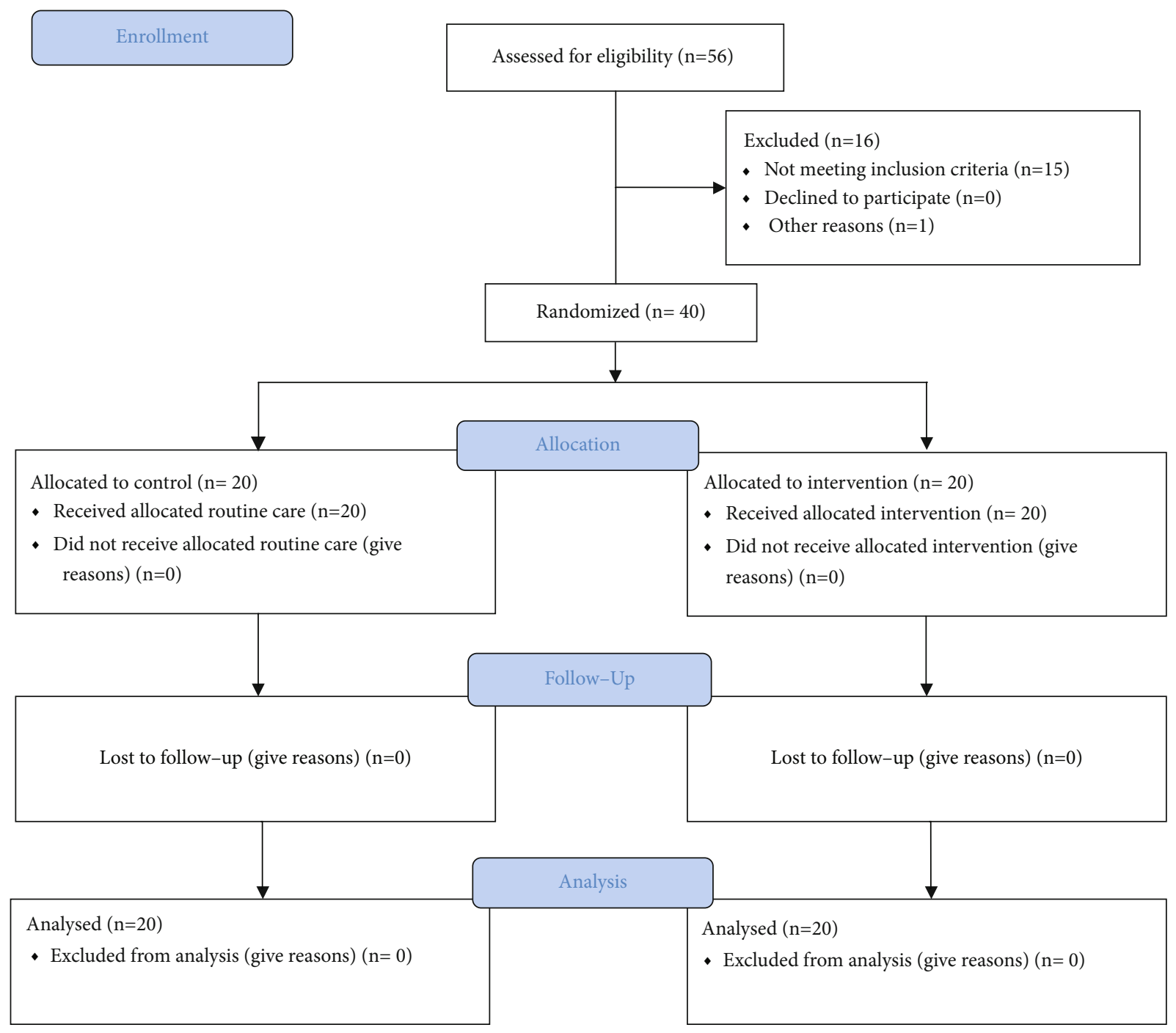

FIgURE 1: Chart of participant's enrollment and procedure.

The total score of the questionnaire is obtained by adding the score of the items. Higher scores indicate the high intensity of insomnia. The reliability of Persian version in Chehri et al.'s had good reliability and validity $(\mathrm{CVR}=0.77$ and Cronbach's alpha 0.84) [21,22].

2.6. The General Health Questionnaire (GHQ-28). The 28-question form of the General Health Questionnaire (GHQ-28) was developed by Goldberg and Hiller in 1979. GHQ-28 scoring is from zero to 3 . If the total score of the questionnaire is more than 23 , it indicates a disorder in general health. Cronbach's alpha coefficient of the Persian version of the questionnaire in the study of Shayan et al. was reported to be 0.90 [23]. We used the GHQ-28 questionnaire to find eligible people, and this questionnaire was used only once in the registration phase.

2.7. Statistical Methods. Data were analyzed using SPSS software version 16 . The normality of the data was examined using the Kolmogorov-Smirnov test; all variables had a normal distribution. To compare the demographic characteristics, we used the chi-square test, and to compare the sleep quality and the insomnia severity between the two groups, we used independent $t$-tests. The effect of group, time, and interaction between time and group was determined using the repeated measure ANOVA test. $P<0.05$ was considered significant statistical level.

\section{Result}

3.1. Baseline Data. The mean age of women in the intervention group was 26.65 years, and in the control group was 28.55 years; the mean gestational age was 18.60 in the intervention group and 18.20 in the control group. Comparing the gestational age and age of women between the two groups was not statistically significant $(P=0.15)$. The results of Table 2 show that the comparison of individual profile 
TABLE 1: Description of the content of spiritual counseling sessions.

(i) Welcome

(ii) Aims and objectives

(iii) Pretest with Insomnia Severity Index (ISI) and the Pittsburgh Sleep Quality Index (PSQI)

Session 1 (iv) Defining of sleep, sleep quality, and preparing a list of stressful situations that affected sleep during pregnancy

(v) Discussing the important of sleep during pregnancy

(vi) Discussing the effect of stress on the body and sleep

(vii) Educating sleep health program and life style modification for managing sleep problems during pregnancy

(viii) Homework: writing the common pregnancy sleep problems and solutions

(i) Encouraging to express their common pregnancy sleep problems and reviewing the solutions

(ii) Discussing one's attitude towards spiritual issues, the role of God, and religion in her life

Session 2 (iii) Discussing one's experiences of reading the Qur'an and listening to the sound of the Qur'an in her calming and the soul

(iv) Homework: writing the spiritual aspects of the pregnancy

(i) Talking about the spiritual aspects of pregnancy, childbearing, and God's reward for pregnant women

(ii) Reciting of verses and hadiths about sleep and the role of sleep comfortably in peace of mind

(iii) Creating a spiritual environment with listening to the voice of the Quran in Surah Ar-Rahman (with the voice of

Session 3 Abdul Basit) for $5 \mathrm{~min}$

(iv) Discussing of one's feels after listening to the voice of the Quran

(v) Homework: listening to the voice of the Quran and relax their muscles with deep breathing for getting rid of the stress/twice daily for 10 to 15 minutes

(i) Reviewing the content of previous sessions and summarize members' opinions

(ii) Blessings of God and the role of it in reinterpreting concept of pregnancy and overcoming the worrisome symptoms of pregnancy

Session 4 (iii) Discussing of prayer therapy to reduce the worries, get rid of some symptoms of pregnancy and to increase hope, resilience

(iv) Homework: encouraging to create a daily spiritual space of time or place at home

(v) Writing the experience of participating in religious programs or doing spiritual issues

(i) Discussing one's experiences of participating in religious programs or doing spiritual issues

(ii) Discussing on the role of charity, trust, recourse, patience, kindness, and forgiveness in managing emotions and life stressors

(iii) Discussing on the role of gratitude to God in enduring hardships, increasing hope and self-confidence, adapting, and

Session 5 accepting the situation

(iv) Encouraging to strengthen one's inner hope, locus of control, and resilience

(v) Reciting the verses of Surah Maryam with the voices of the volunteer members with focusing its meaning

(vi) Homework: writing down situations that make you feel guilty or angry about yourself or others

(i) Discussing on one's experiences of forgiveness in life and anger towards the guilty

(ii) Discussing the meaning of life with the birth of a baby

Session 6 (iii) Listening to "nature's music" the sound of birds, rivers and waterfalls with illustration hugging her baby

(iv) Encouraging to participate in charity, kindness, and forgiveness

(v) Homework: writing some strategies to cope with stress and improving sleep quality

(i) Reviewing their strategies to cope with stress and improving sleep quality

(ii) Designing a play of a stressful situation, charting a table of tensions, the severity of tension, behavior and attitudes,

Session 7 satisfaction with the coping method employed in those situations, and simple suggestions to cope with stress

(iii) Homework: listening to the voice of the Quran and relax their muscles with deep breathing for getting rid of the stress/twice daily for 10 to 15 minutes

(i) Reviewing the content of previous sessions and summarize members' opinions

Session 8

(ii) Discussing one's experiences of spiritual self-awareness, problem-solving skills with a spiritual approach

variables in the two groups was not statistically significant, so the two groups were homogeneous $(P=0.31)$ (Table 2).

3.2. Sleep Quality. In the counselling group, the mean (SD) of a total score of sleep quality before the intervention was
9.45 (2.30) which decreased to 5.40 (1.56) in 36 weeks of gestational age. While the control group was increased from $9.26(2.15)$ to $11.47(1.54)$ at the same time. In the counselling group based on the repeated measure ANOVA test, the total mean score of sleep quality and its components 
TABLE 2: Comparison of the frequency distribution of demographic characteristics between two study groups.

\begin{tabular}{|c|c|c|c|c|c|}
\hline \multirow{2}{*}{ Social and midwifery characteristics } & \multicolumn{2}{|c|}{ Intervention } & \multicolumn{2}{|r|}{ Control } & \multirow[t]{2}{*}{$P$ value* } \\
\hline & $N$ & $\%$ & $N$ & $\%$ & \\
\hline \multicolumn{6}{|l|}{ Education } \\
\hline Guidance & 3 & 15 & 7 & 35 & \multirow{3}{*}{0.31} \\
\hline Diploma & 13 & 65 & 9 & 45 & \\
\hline Academic & 4 & 20 & 4 & 20 & \\
\hline \multicolumn{6}{|l|}{ Job } \\
\hline Unemployed & 20 & 100 & 19 & 95 & \multirow[t]{2}{*}{0.50} \\
\hline Employed & 0 & 0 & 1 & 5 & \\
\hline \multicolumn{6}{|l|}{ Family monthly income } \\
\hline Not enough & 2 & 10 & 1 & 5 & \multirow{3}{*}{0.42} \\
\hline To some degree enough & 7 & 35 & 11 & 55 & \\
\hline Sufficient & 11 & 55 & 8 & 40 & \\
\hline \multicolumn{6}{|l|}{ Housing status } \\
\hline Owner & 8 & 40 & 7 & 35 & \multirow[t]{2}{*}{0.74} \\
\hline Rented & 12 & 60 & 13 & 65 & \\
\hline \multicolumn{6}{|l|}{ Gravid } \\
\hline First pregnancy & 10 & 50 & 10 & 50 & \multirow[t]{2}{*}{1} \\
\hline$\geq$ two pregnancy & 10 & 50 & 10 & 50 & \\
\hline
\end{tabular}

${ }^{*}$ Chi-square test.

significantly decreased after the intervention compared to the control group $(P=0.001)$. Furthermore, neither participant in the two groups used the sleep pills to control their sleep disorder (Table 3).

A comparison of the mean scores of sleep quality between the two groups in three time periods is shown in Figure 2.

3.3. Insomnia Severity. In the counselling group, the mean total score of insomnia severity after the intervention was decreased in 36 weeks of gestational age compared to the control group $(P=0.001)$. Based on the repeated measure ANOVA test, the total mean score of insomnia severity in the second and third trimesters decreased compared to the control group. The observed differences between the two groups were statistically significant $(P=0.001)$ The effect size of intervention ("Eta" score) at the end of the followup period was about 0.66 ; it shows that counselling with a spiritual content was associated with a $66 \%$ reduction in the severity of insomnia (Table 4).

A comparison of the mean scores of insomnia severity between the two groups in three time periods is shown in Figure 3.

\section{Discussion}

The study is aimed at determining the efficacy of spiritual content counselling on improving the sleep quality and insomnia severity of pregnant women. The results of the present study showed that counselling with spiritual content was effective in improving the sleep quality in pregnant women. The efficacy of the intervention continued to the second and third trimesters. Although no available studies were showing the effect of spiritual content counselling on sleep quality during pregnancy, the positive effects of spiritual counselling on improving sleep quality have been reported in the nonpregnant research community. Taheri et al. (2013) showed that after spiritual care based on the "Ghalb Salim" nursing model, the mean score of sleep quality in patients with coronary artery disease had a statistically significant difference in comparison with the control group [9]. Soheyli et al. (2013) reported a similar result [24]. In the present study, spiritual content counselling had a significant effect on all areas of sleep quality, except for sleep efficiency. However, in the above studies, an improvement was observed in all areas of sleep quality.

Some studies have emphasized various beliefs about the biological and psychological effects of spiritual-oriented counselling. Spirituality, as a powerful factor, can influence one's attitude, cognition, and behaviour, and as a mediator, can affect one's thinking and evaluation process [19, 25]. In the Qur'an, God describes sleep with the word "Noum" and says in the ninth verse of Surah Naba "We have made your sleep a source of peace, the mentally and physically rejuvenation, and to rebuild the worn-out limbs and relieve any fatigue or discomfort". From the content of the tenth verse of Surah Anfal, it is clear that sleep causes the elimination of fear and the feeling of security. This shows that sleep and a sense of security complete each other [26].

The peace of mind caused by religious activities (e.g., listening to religious sounds) has been emphasized to reduce anxiety during pregnancy [27]. Peace of mind and healthy sleep are essential in exuding positive hormones from the brain, improving protein metabolism, increasing the function of the immune system, and reducing the stress reactions in the body [19]. According to our result, another positive 
TABLE 3: The comparison of sleep quality between the two groups in three stage (preintervention, 28, and 36 weeks of gestation).

\begin{tabular}{|c|c|c|c|c|c|c|c|c|c|c|}
\hline \multirow{2}{*}{ Variables } & \multirow{2}{*}{ Time } & \multicolumn{2}{|c|}{ Intervention } & \multicolumn{2}{|c|}{ Control } & \multirow[t]{2}{*}{${ }^{* *} P$ value } & \multirow[t]{2}{*}{$F$} & \multirow[t]{2}{*}{ Eta } & \multirow{2}{*}{$\mathrm{MD}^{* * *}$} & \multirow{2}{*}{${ }^{*} P$ value } \\
\hline & & Mean & $\mathrm{SD}$ & Mean & $\mathrm{SD}$ & & & & & \\
\hline \multirow{3}{*}{$\begin{array}{l}\text { Sleep quality } \\
\text { Total score }\end{array}$} & Baseline & 9.45 & 2.30 & 9.26 & 2.15 & 0.75 & & & & \\
\hline & $28^{\text {th }} \mathrm{w}$ & 5.70 & 1.55 & 11.63 & 2.06 & 0.001 & 66.90 & 0.64 & -3.93 & 0.001 \\
\hline & $36^{\text {th }} \mathrm{w}$ & 5.40 & 1.56 & 11.47 & 1.54 & 0.001 & & & & \\
\hline \multirow{3}{*}{ Subjective sleep quality } & Baseline & 0.8 & 0.69 & 1.00 & 0.66 & 0.36 & & & & \\
\hline & $28^{\text {th }} \mathrm{w}$ & 0.3 & 0.47 & 1.58 & 0.69 & 0.001 & 19.90 & 0.35 & -0.96 & 0.001 \\
\hline & $36^{\text {th }} \mathrm{w}$ & 0.1 & 0.30 & 1.53 & 0.69 & 0.001 & & & & \\
\hline \multirow{3}{*}{ Sleep latency } & Baseline & 1.75 & 0.63 & 1.57 & 0.60 & 0.39 & & & & \\
\hline & $28^{\text {th }} \mathrm{w}$ & 0.85 & 0.58 & 2.05 & 0.62 & 0.001 & 37.49 & 0.50 & -0.79 & 0.001 \\
\hline & $36^{\text {th }} \mathrm{w}$ & 0.80 & 0.41 & 2.15 & 0.60 & 0.001 & & & & \\
\hline \multirow{3}{*}{ Sleep duration } & Baseline & 0.45 & 0.82 & 0.52 & 0.84 & 0.77 & & & & \\
\hline & $28^{\text {th }} \mathrm{w}$ & 0.1 & 0.30 & 0.73 & 0.87 & 0.001 & 7.09 & 0.16 & -0.51 & 0.002 \\
\hline & $36^{\text {th }} \mathrm{w}$ & 0 & 0 & 0.84 & 0.83 & 0.001 & & & & \\
\hline \multirow{3}{*}{ Sleep efficiency } & Baseline & 3.0 & 0.01 & 2.94 & 0.22 & 0.31 & & & & \\
\hline & $28^{\text {th }} \mathrm{w}$ & 3.0 & 0.01 & 2.89 & 0.45 & 0.31 & 1.05 & 0.02 & 0.08 & 0.31 \\
\hline & $36^{\text {th }} \mathrm{w}$ & 3.0 & 0.01 & 2.89 & 0.45 & 0.31 & & & & \\
\hline \multirow{3}{*}{ Sleep disturbances } & Baseline & 0.90 & 0.30 & 0.89 & 0.31 & 0.95 & & & & \\
\hline & $28^{\text {th }} \mathrm{w}$ & 0.55 & 0.51 & 0.94 & 0.22 & 0.004 & 6.07 & 0.14 & -0.26 & 0.004 \\
\hline & $36^{\text {th }} \mathrm{w}$ & 0.60 & 0.50 & 1.0 & 0.01 & 0.001 & & & & \\
\hline \multirow{3}{*}{ Daytime dysfunction } & Baseline & 0.7 & 0.57 & 0.78 & 0.71 & 0.66 & & & & \\
\hline & $28^{\text {th }} \mathrm{w}$ & 0.3 & 0.47 & 1.57 & 0.6 & 0.001 & 24.46 & 0.41 & -0.79 & 0.001 \\
\hline & $36^{\text {th }} \mathrm{w}$ & 0.2 & 0.41 & 1.21 & 0.53 & 0.001 & & & & \\
\hline \multirow{3}{*}{ Sleeping medication } & Baseline & 0 & 0 & 0 & 0 & 0 & & & & \\
\hline & $28^{\text {th }} \mathrm{w}$ & 0 & 0 & 0 & 0 & 0 & - & - & - & - \\
\hline & $36^{\text {th }} \mathrm{w}$ & 0 & 0 & 0 & 0 & 0 & & & & \\
\hline
\end{tabular}

** Independent $t$ test; ${ }^{*}$ repeated measures ANOVA; ${ }^{* * *}$ mean diff.

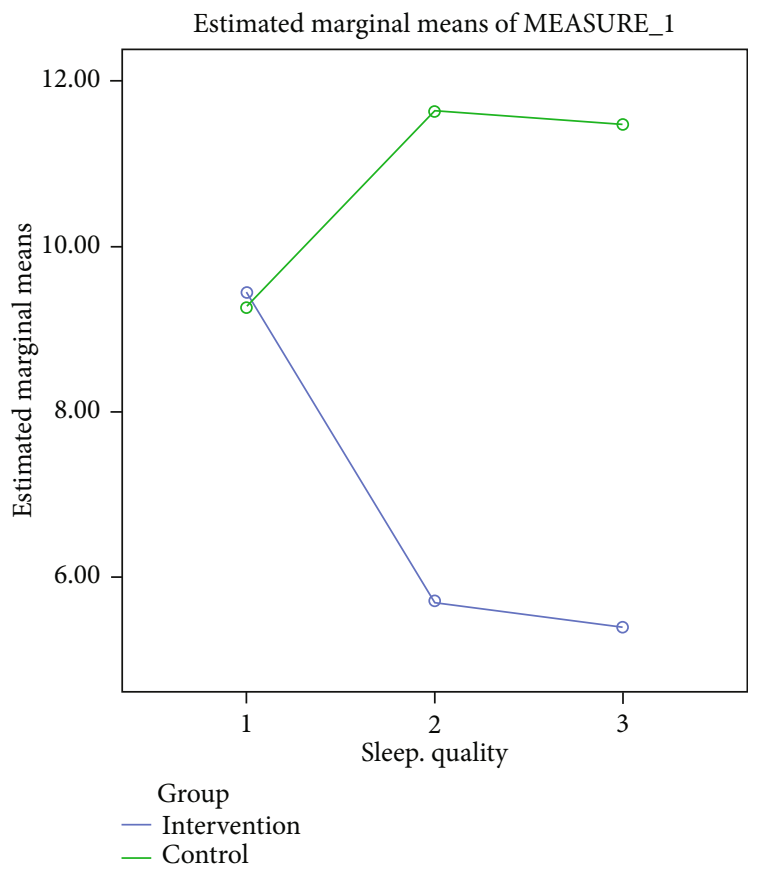

Figure 2: Chart of sleep quality in two groups. effect of spirituality-based counselling is the improvement of sleep quality during pregnancy, from which the providers can benefit in the form of maternity care service packages.

In the present study, we used religious sounds during relaxation and meditation exercises, which had similar effects on the results of mindfulness and relaxation-based interventions [28]. Mindfulness strategies play a role in reducing stress and regulating emotional response. For instance, yoga leads to communication with higher and superior powers. The results of the present study show that spiritual counselling as well as the two interventions listed are useful in reducing the cognitive and physical arousal associated with insomnia in pregnancy.

In the present study, part of the intervention focused on sleep health education and identifying sleep stimuli. It seems that increasing spiritual attention, along with health advice and behaviour modification, can have a positive effect on sleep quality.

The results of the present study showed that after the intervention, the mean score of the areas of subjective sleep quality, sleep latency, sleep duration, sleep disturbances, use of sleeping medication, and daytime dysfunction in the intervention group decreased significantly. In contrast, the score of these areas in the control group had a significant 
TABLE 4: The comparison of insomnia severity between the two groups in three stage (preintervention, 28, and 36 weeks of gestation).

\begin{tabular}{|c|c|c|c|c|c|c|c|c|c|c|}
\hline \multirow{2}{*}{ Variables } & \multirow{2}{*}{ Time } & \multicolumn{2}{|c|}{ Intervention } & \multicolumn{2}{|c|}{ Control } & \multirow[t]{2}{*}{${ }^{* *} P$ value } & \multirow[t]{2}{*}{$F$} & \multirow[t]{2}{*}{ Eta } & \multirow[t]{2}{*}{ Mean diff } & \multirow[t]{2}{*}{${ }^{*} P$ value } \\
\hline & & Mean & $\mathrm{SD}$ & Mean & $\mathrm{SD}$ & & & & & \\
\hline \multirow{3}{*}{ Insomnia severity } & Baseline & 7.05 & 4.29 & 7.05 & 4.19 & 0.99 & & & & \\
\hline & $28^{\text {th }} \mathrm{w}$ & 2.55 & 1.60 & 11.47 & 3.23 & 0.001 & 8.79 & 0.66 & -6.66 & 0.001 \\
\hline & $36^{\text {th }} \mathrm{w}$ & 1.55 & 0.94 & 12.63 & 3.7 & 0.001 & & & & \\
\hline
\end{tabular}

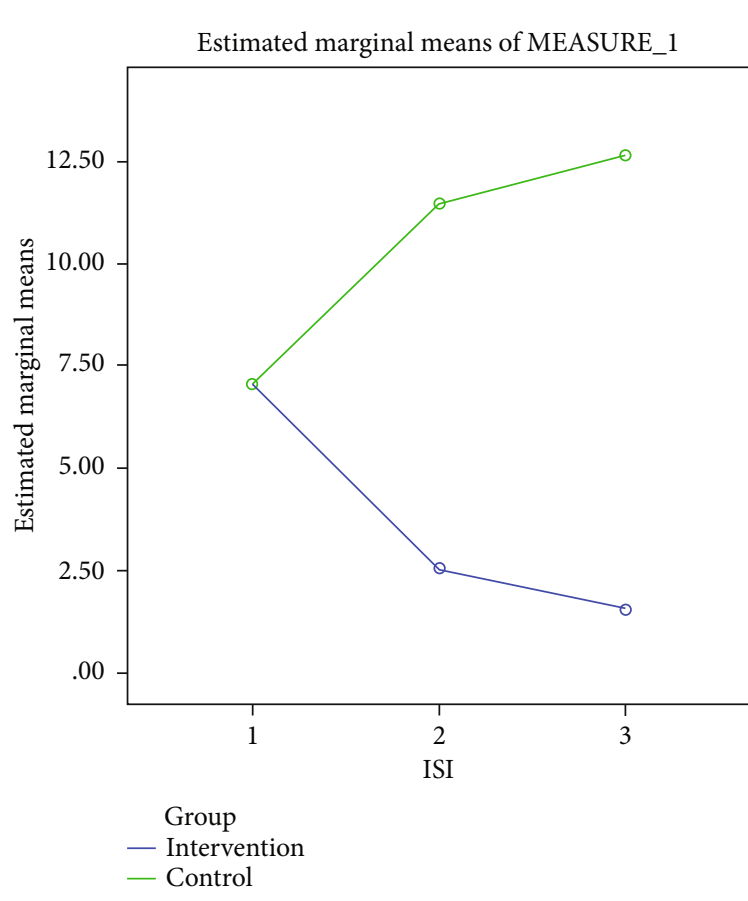

FIgURe 3: Chart of Insomnia Severity Index (ISI) in two groups.

increase. Comparing the scores of sleep efficiency in the two groups was not statistically significant. Also, none of the participants in either group used medication to control sleep disorders. In a clinical trial study, Felder et al. examined the efficacy of digital counselling using the cognitivebehavioural approach in treating the symptoms of insomnia among 208 pregnant women with a mean gestational age of 17 weeks. The intervention group received 6 sessions of online counselling per week in 5 areas (sleep restriction, stimulus control, cognitive therapy, relaxation techniques, and sleep hygiene training), and the control group received routine care. The follow-up period was 10 weeks after the end of the intervention. The results showed that the mean score of sleep quality and habitual sleep efficiency in the intervention group was significantly lower than the control group, while the mean score of sleep duration did not differ [29]. The results of the above study were consistent with the results of the present study in terms of overall sleep quality score, but not in terms of habitual sleep efficiency and sleep duration, which can be due to easy access to an online consultant.

The results of a review of 24 articles show that the mean score of sleep quality during pregnancy was 6.07. About $45.7 \%$ of pregnant women experience some degree of diminished sleep quality during pregnancy. However, changes in sleep quality score increase in the third trimester were 1.68 times higher than in the second trimester [2]. In the present study, the changes in the overall score of sleep quality in the control group were consistent with the results of the above study, so that the mean score of sleep quality of pregnant women increased from 9.26 in the first quarter to 11.47 in the third quarter. However, spiritual-oriented counselling reduced the overall score of sleep quality from 9.45 to 5.40. Therefore, using the present approach can be considered as a basis for improving sleep quality during pregnancy.

Religious activities can be a social determining factor in the state of sleep. So those who engage in religious activity are more likely to have a healthy sleep than those who do not. Based on the conceptual model proposed in a study, religious conflict may increase with hope, better support for mental health, a healthy lifestyle, reduced stress, healthier physiological function, limited substance use, and exposure to stress leading to healthy sleep [30]. However, conflicting results have also been reported. For example, Gillum (2013) showed that there was no significant relationship between participation in religious ceremonies in people over 40 years of age and sleep disorders [30], the results of which are inconsistent with the results of the present study because of the difference in the research population.

The results of the present study showed that spiritual content counselling was effective in improving the severity of insomnia in pregnant women. In the intervention group, the mean score of insomnia severity before the intervention decreased from 7.05 to 1.55 in the third trimester, and in the control group, it increased from 7.05 to 12.63 . Comparing the mean scores in the three-time periods between the two groups was significant. So far, many interventions have been made to treat insomnia in pregnant women, but due to the adverse effects of drugs in pregnancy, researchers are trying to focus on nonpharmacological methods. The results of the present study are consistent with the results of some nonpharmacological interventions. As Jalal Marvi et al. showed (2019), distance education has positive effects on improving the intensity of insomnia in pregnant women [31]. In another study, Felder et al. (2020) showed that the implementation of 6 sessions of digital counselling based on the cognitive-behavioural approach could alleviate the intensity of insomnia in pregnant women [32]. Tomfohr-Madsen et al. reported similar results in 2017. In their study, holding 5 group counselling sessions based on the cognitivebehavioural approach had positive effects on alleviating the intensity of insomnia in pregnant women in the second trimester of pregnancy [33]. The findings of our study showed that integration of sleep hygiene training with spiritual orders can be as effective as other interventions. 
It can be argued that some cognitive patterns, psychological characteristics, and behavioural patterns created by spiritual-oriented methods lead to improved health and improved physiological function of the body, followed by high psychological resistance in inappropriate physical and social situations. Accordingly, religious and spiritual practices lead to increased tolerance, patience, self-control, positivism, satisfaction, emotional control, optimism, selfefficacy (based on trust in God's blessing), altruism, kindness, and love [19]. The skill of "spiritual resilience" is another technique of spiritual counselling that encourages the audience to overcome physical and mental problems in a variety of ways.

4.1. Strengths of Study. All the principles of control trial studies were observed in this study, and we do not have a loss of following in participants. Data collection tools were standard, and psychometric properties of the Persian form of the questionnaires have been evaluated based on Iranian culture.

4.2. Limitations. Data collection was performed using a selfreport questionnaire, and all participants were Muslim which should be considered in the generalizability of our findings. Also, the sample size was small, and the meeting was held without the attending of their spouse. Future studies with large sample sizes and is based on other cultures are required for a better conclusion.

4.3. Implications for Practice and/or Policy. In the present study, the effects of spiritual content counselling were not significant on habitual sleep efficiency. Given the importance of sleep in the physical and mental health of pregnant mothers, it is recommended that other psychological approaches be conducted in this field.

\section{Conclusion}

The results of the present study showed that counselling with spiritual content could effectively ameliorate sleep quality and reduce insomnia severity in pregnant mothers. Also, improving sleep quality continued until the third trimester of pregnancy. However, the control group had worse sleep quality with increasing gestational age than the intervention group. It seems that the approach of the present study is an acceptable basis to design intervention programs in this field that can be considered by researchers, consultants, and midwives.

\section{Abbreviations}

PSQI: The Pittsburgh Sleep Quality Index

ISI: The Insomnia Severity Index.

\section{Data Availability}

The data sets used and analyzed during the current study are available from the corresponding author on reasonable request.

\section{Ethical Approval}

This article was part of MSc thesis and approved by the Ethics Committee of the Vice Chancellor for Research of Zanjan University of Medical Sciences, Iran, with the approval IR.ZUMS.REC.1398.153.

\section{Consent}

The objectives of the study were explained to the participating mothers and all participants signed written informed consent forms. Subjects could leave the study at any time, and they were also assured about the confidentiality of their data.

\section{Disclosure}

A preprint of the manuscript has previously been published in "Research square" [Ref.no. doi:10.21203/rs.3.rs-1025124/ v1] [34].

\section{Conflicts of Interest}

No potential conflict of interest relevant to this article was reported.

\section{Authors' Contributions}

This study was a part of MSc thesis of N.M. The conception, design of the study, and data collection process were undertaken by N.M. A. M was the supervisor, and S. Z was the adviser. They are also contributed to the design of the study and reporting of the result. Analysis, interpretation, and reporting were supervised by A. M and N.M. All authors contributed in drafting the article, revising it, and in agreement with the final version of the manuscript to be submitted to the journal, and they also met the criteria of authorship.

\section{Acknowledgments}

We would like to thank Zanjan University's vice-chancellor of education and vice-chancellor of research and technology for their financial support to carry out the study (the code "A-11-344-9"). We would like to thank the staff of the health centres of Abhar for their collaboration and mothers for their participation. This article was part of MSc thesis and funded by the Research Deputy of Zanjan University of Medical Sciences, Iran, with the approval number (the code “A-11-344-9").

\section{References}

[1] I. D. Sedov, E. E. Cameron, S. Madigan, and L. M. TomfohrMadsen, "Sleep quality during pregnancy: a meta-analysis," Sleep Medicine Reviews, vol. 38, pp. 168-176, 2018.

[2] M. Kaveh, "Sleep disorders in iranian pregnant women: a systematic review and meta-analysis," International Journal of Pharmaceutical and Biological Science Archive, vol. 7, no. $6,2019$. 
[3] A. Crivello, P. Barsocchi, M. Girolami, and F. Palumbo, "The meaning of sleep quality: a survey of available technologies," IEEE access, vol. 7, pp. 167374-167390, 2019.

[4] Z. Yang, Z. Zhu, C. Wang, F. Zhang, and H. Zeng, “Association between adverse perinatal outcomes and sleep disturbances during pregnancy: a systematic review and meta-analysis," The Journal of Maternal-Fetal \& Neonatal Medicine, vol. 35, no. 1, pp. 166-174, 2022.

[5] C. L. Dixon, "Sleep deprivation \& pregnancy related risks," International Journal of Childbirth Education, vol. 29, no. 3, 2014.

[6] C. H. Won, "Sleeping for two: the great paradox of sleep in pregnancy," Journal of Clinical Sleep Medicine, vol. 11, no. 6, pp. 593-594, 2015.

[7] Y.-H. Lin, C. Chen, X. Zhao et al., "Efficacy and safety of Banxia formulae for insomnia: a systematic review and meta-analysis of high-quality randomized controlled trials," Evidence-based Complementary and Alternative Medicine, vol. 2021, Article ID 8833168, 18 pages, 2021.

[8] F. Afrasiabian, M. Mirabzadeh Ardakani, K. Rahmani et al., "Aloysia citriodora Palau (lemon verbena) for insomnia patients: a randomized, double-blind, placebo-controlled clinical trial of efficacy and safety," Phytotherapy Research, vol. 33, no. 2, pp. 350-359, 2019.

[9] Z. S. Taheri, M. A. Zandi, and A. Ebadi, "The effect of spiritual care based on Ghalbe Salim model on the sleep quality of the patients with coronary artery disease," Iranian Journal of Critical Care Nursing (IJCCN), vol. 7, no. 2, pp. 92101, 2014.

[10] E. Ghadampour, S. Roshannia, and N. Rezaei, "Measuring the effectiveness of group spirituality therapy on sleep quality and mental toughness in elderly subjects in Khorramabad," Journal of Gerontology (joge), vol. 3, no. 2, pp. 31-39, 2018.

[11] N. Gorji, R. Moeini, and S. A. Mozaffarpur, "On the therapeutic applications of music therapy in Persian medicine," Traditional and Integrative Medicine, vol. 6, 2021.

[12] H. G. Koenig, "Research on religion, spirituality, and mental health: a review," Canadian Journal of Psychiatry, vol. 54, no. 5, pp. 283-291, 2009.

[13] M. Haghighat, M. Mirghafourvand, S. Mohammad-AlizadehCharandabi, J. Malakouti, and M. Erfani, "The effect of spiritual counseling on stress and anxiety in pregnancy: a randomized controlled clinical trial," Iranian Red Crescent Medical Journal, vol. In Press, no. In Press, 2018.

[14] S. Mohamadirizi, M. Mohamadirizi, S. Mohamadirizi, and F. Mahmoodi, "The effect of religious-spiritual support on childbirth self-efficacy," Journal of Education Health Promotion, vol. 7, p. 14, 2018.

[15] S. Tayebi, Z. Montaseri, M. Edraki, and M. Akbarzadeh, "Relationship between mothers' spiritual health scores with newborns' physical development indices and physiologic parameters in Hazrat Zeinab training hospital," Iranian Journal of Neonatology IJN, vol. 11, no. 1, pp. 51-59, 2020.

[16] A. L. Rodriguez, J. Arcara, J. Deardorff, and A. M. Gomez, "The association between religiosity and pregnancy acceptability among Latino/a young adults: does generational status matter?," Culture, Health \& Sexuality, vol. 22, no. 2, pp. 184-200, 2020.

[17] M. M. Malikzadegan Aqdas, A. Hassan, and H. Hamid, "The effect of relaxation exercises on sleep disorders in the third tri- mester of pregnancy," Iranian Journal of Nursing, vol. 23, pp. 52-58, 2010.

[18] M. Bolhari, Spiritual Skills Training, M. Mirzaie and Z. Ghaempanah, Eds., Tehran University of Medical Sciences, 2013.

[19] M. Asad Zandi, Analysis of nursing concepts and theories (along with the presentation of the Salim Heart Model), Heidary, Tehran, 2014.

[20] N. Mohammad Gholi Mezerji, P. Naseri, Z. Omraninezhad, and Z. Shayan, "The reliability and validity of the Persian version of Pittsburgh sleep quality index in Iranian people," Avicenna Journal of Neuro Psycho Physiology, vol. 4, no. 3, pp. 95-102, 2017.

[21] K. R. Timpano, J. Y. Carbonella, R. A. Bernert, and N. B. Schmidt, "Obsessive compulsive symptoms and sleep difficulties: exploring the unique relationship between insomnia and obsessions," Journal of Psychiatric Research, vol. 57, pp. 101107, 2014.

[22] A. Chehri, N. Goldaste, S. Ahmadi, H. Khazaie, and A. Jalali, "Psychometric properties of insomnia severity index in Iranian adolescents," Sleep Science, vol. 14, no. 2, p. 101, 2021.

[23] Z. Shayan, Z. Pourmovahed, F. Najafipour, A. M. Abdoli, F. Mohebpour, and S. Najafipour, "Factor structure of the General Health Questionnaire-28 (GHQ-28) from infertile women attending the Yazd Research and Clinical Center for Infertility," International journal of reproductive biomedicine, vol. 13, no. 12, p. 801, 2015.

[24] M. Soheyli, A. Rahimi, and A. Chavoshi, "Effect of sleep based on Islamic pattern on the quality of sleep in mustard gas chemical casualties," Iranian Journal of War \& Public Health, vol. 6, no. 4, pp. 151-156, 2014.

[25] A. Srivastava and A. Krishna, "Psycho-physiological mechanism of spirituality-health relationship: theoretical conceptualisation," Journal of Indian Health Psychology, vol. 2, no. 1, pp. 1-9, 2013.

[26] S. Wahyuni, T. Rahayu, H. Distinarista, A. Y. Wuriningsih, and S. D. Nofitasari, "Spiritual intervention and thermal stimulation in pregnant women with back pain," Indian Journal of Public Health Research \& Development, vol. 10, no. 8, pp. 2746-2750, 2019.

[27] B. Khodakarami, F. Golalizadeh Bibalan, F. Soltani, A. Soltanian, and H. Mohagheghi, "Impact of a counseling program on depression, anxiety, stress, and spiritual intelligence in pregnant women," Journal of Midwifery and Reproductive Health, vol. 5, no. 2, pp. 858-866, 2017.

[28] V. Bacaro, F. Benz, A. Pappaccogli et al., "Interventions for sleep problems during pregnancy: a systematic review," Sleep Medicine Reviews, vol. 50, article 101234, 2020.

[29] T. D. Hill, R. Deangelis, and C. G. Ellison, "Religious involvement as a social determinant of sleep: an initial review and conceptual model," Sleep Health, vol. 4, no. 4, pp. 325-330, 2018.

[30] R. F. Gillum, "Religious behavior, sleep quantity, sleep quality, and sleep disorders in American adults," The Journal of Behavioral Health Services \& Research, vol. 40, no. 1, p. 133, 2013.

[31] F. Jalal Marvi et al., "Comparing the effect of training based on continuous care model and telehealth on severity of insomnia in pregnant women," Journal of North Khorasan University of Medical Sciences, vol. 11, no. 3, pp. 38-45, 2019.

[32] J. N. Felder, E. S. Epel, J. Neuhaus, A. D. Krystal, and A. A. Prather, "Efficacy of digital cognitive behavioral therapy for 
the treatment of insomnia symptoms among pregnant women: a randomized clinical trial," JAMA Psychiatry, vol. 77, no. 5, pp. 484-492, 2020.

[33] L. M. Tomfohr-Madsen, Z. M. Clayborne, C. R. Rouleau, and T. S. Campbell, "Sleeping for two: an open-pilot study of cognitive behavioral therapy for insomnia in pregnancy," Behavioral Sleep Medicine, vol. 15, no. 5, pp. 377-393, 2017.

[34] https://www.researchsquare.com/article/rs-1025124/v1. 